\title{
Is science losing out in the race for recognition?
}

\author{
Progress is made through the achievements of many who are not singled out for reward.
}

Sir - The dance around the golden Nobel medallion began more than a century ago and is still going strong. Raymond Damadian's public dispute (see "Physician launches public protest over medical Nobel" Nature 425, 648; 2003) should make us ask whether science is best served by a culture obsessed with rankings and winning prizes. The history of the Nobel Prize makes it clear that the medallion is etched with human frailties.

Winning a Nobel Prize is never an automatic process, a reward that comes for having attained a magical level of achievement. Although international in scope, the prize is a Swedish prerogative. Historical study shows that Swedish committee members' own understanding of science affects their recommendations. Their judgements, predilections and interests necessarily enter into their deliberations. Academy physicists had no intention of recognizing Einstein's theories of relativity "even if the whole world demands it". A simple change in the composition of the committee can decide the fate of a candidate.

But even when those involved rise above pettiness and partiality, the task of selecting winners remains exceedingly difficult. Committee members have confessed that often several candidates can be found who equally deserve a prize. Unambiguous, impartial criteria for selecting among several deserving candidates are not at hand. There are no grounds for assuming that the laureates constitute a unique population of the very best in science.

The prizes reward these few individuals when, in reality, achievement arises through a broader spectrum of accomplishment by many talented scientists. Let us not forget that some important branches of science are not addressed by Nobel's testament. Some of the greatest intellectual triumphs of the past century have not been celebrated in Stockholm.

Why then do people venerate the Nobel Prizes? There is no simple answer. The cult of the prize began from the very start; media fascination whipped up speculation and interest. Leaders of national scientific communities willingly climbed on the bandwagon, and over time the number of parties with a stake in maintaining the cult of the prize has grown.

Damadian's campaign to have a share in the prize for his work on developing magnetic resonance imaging (MRI) is a product of a scientific culture based on competition for personal and institutional aggrandizement. Whatever Alfred Nobel might have meant when he set up prizes for those whose work conferred "the greatest benefit on mankind”, he did not have in mind the promotion of narrow professional interests, nor institutional and national boosterism.

Should racing to discovery define the soul of science? Its heritage is far richer than the quest for prizes might suggest. Robert Marc Friedman

Department of History, University of Oslo, Pb. 1008 Blindern, 0315 Oslo, Norway

\section{GM-debate methodology works in the real world}

Sir - Scott Campbell and Ellen Townsend's critique (Nature 425, 559; 2003) of the GM Nation? report makes a big claim: "The methodology was so badly flawed that the data not only failed to support the authors' conclusions, but undermined them". As a social scientist and a member of the steering board for this debate on genetic modification (I write in a personal capacity), I am unimpressed. They misrepresent the nature of the exercise overall and their critique is flawed.

Campbell and Townsend approach the GM Nation? report (www.gmnation.org) as if it were a narrow psychometric study, rather than an account of a multifaceted process of public debate, taking place in real time in a politically contentious field. Owing to this misreading, their analysis is selective and misleading.

For example, in their analysis of data gathered from two of the primary activities involved in this exercise - the nationwide open debates, and the more controlled narrow-but-deep (NBD) focus groups - the authors miss the central point of this twin-track approach. In addition to understanding how far the views expressed by the NBD groups matched those of the inevitably self-selecting participants in the open events, we also wished to follow the evolving views of NBD participants as they acquired more understanding of GM-related issues over a two-week period.

Insights from this exercise contributed towards the steering board's judgements about the likely state of latent, as well as explicitly expressed, public opinion and to our confidence in the robustness of the key findings.

No one would claim that the GM debate was a flawless exercise, though, like others involved, I regard it as time fruitfully spent. It will be and should be evaluated rigorously, not least for lessons that can be learned for the benefit of similar exercises in the future. The Campbell and Townsend critique is not a helpful contribution in this regard. Robin Grove-White

Institute for Environment, Philosophy and Public Policy, Lancaster University, Lancaster LA1 4YW, UK

\section{Citation rate unrelated to journals' impact factors}

Sir - As rightly pointed out by David Colquhoun in Correspondence "Challenging the tyranny of impact factors" (Nature 423, 479; 2003) — the citation rate of the individual paper is essentially uncorrelated to the impact factor of the journal in which it was published.

Adding insult to injury, the impact factors of many journals also change over time. To quantify this further, I selected journals whose impact factor is available from 1992 to 2001 from Roman Woelfel's website (http://staff-www.uni-marburg.de/ $\sim$ woelfel/impact.html). When I compared data for more than 3,000 journals, I found that $26.8 \%$ of them had at least doubled their impact factors over this time period, whereas $1.8 \%$ had decreased by up to half.

A few journals (1.9\%) had increased their impact factor more than tenfold by 2001 , although most of these had very low impact factors to start with. Among the most notable increases from 1992 to 2001: Behavioral and Brain Sciences from 0.30 to 17.31; CA: A Cancer Journal for Clinicians from 5.02 to 35.93; Current Opinion in Immunology from 2.16 to 13.72; The Journal of the American Medical Association from 5.56 to 17.57 .

Notable decreases included the Federation of American Societies for Experimental Biology's FASEB Journal from 18.21 to 8.82 and Quarterly Reviews of Biophysics from 13.0 to 3.94 .

When scientists are being evaluated on the basis of the impact factors of the journals in which they publish, such distortions should be kept in mind.

\section{A. A. Waheed}

Metabolic Diseases Branch, National Institute of

Diabetes and Digestive and Kidney Diseases,

National Institutes of Health, Bethesda,

Maryland 20892-1802, USA 